\title{
T-DM1 for advanced breast cancer after multi-anti-HER2-target therapy: a case report
}

\author{
Langshuang Sun ${ }^{1}$, Lu Gan $^{1 \#}$, Tao Wang ${ }^{2 \#}$ \\ ${ }^{1}$ Department of Oncology, The First Affiliated Hospital of Chongqing Medical University, Chongqing, China; ${ }^{2}$ Department of Breast Oncology, \\ The Fifth Medical Center of Chinese PLA General Hospital, Beijing, China \\ \#These authors contributed equally to this work. \\ Correspondence to: Lu Gan. Department of Oncology, The First Affiliated Hospital of Chongqing Medical University, No.1 Youyi Road, Yuzhong \\ District, Chongqing 400016, China. Email: ganlu99@sina.com; Tao Wang. Department of Breast Oncology, The Fifth Medical Center of Chinese \\ PLA General Hospital, No. 8 Dongda Street, Fengtai District, Beijing 100071, China. Email: wangtao733073@163.com.
}

\begin{abstract}
We report a case of human epidermal growth factor receptor 2 positive (HER2+) breast cancer progressing to multiple metastases during several lines of anti-Her2 targeted therapy and chemotherapy, showing responses to TDM-1 and receiving clinical complete remission. The patient was diagnosed with HER2 + stage IIA invasive carcinoma of the left breast. The tumor progressed with skin invasion after neoadjuvant chemotherapy and continued progressing with sternum metastasis after trastuzumab-contained salvage treatment and dual-targeted therapy combined with chemotherapy. Afterward, the next-generation sequencing (NGS) test detected a PIK3CA mutation and the amplification of ERBB2. A third-line salvage therapy using everolimus and trastuzumab combined with chemotherapy consequently resulted in stable disease. After the therapy of TDM-1, the tumor received a durable complete clinical remission (cCR) that was maintained for 30 months. The regimen was initially TDM1 once 3 weeks for 19 months, then modified to once 4 weeks after the cCR statement lasted for 1year, and discontinued after another 10 months. During the medication, the patient regularly underwent the PET-CT every 3 to 4 months and was still disease-free until this report. This case delivers a view that keeping anti-HER2 therapy and adjusting regimens precisely according to gene sequencing can bring advanced HER2+ breast cancer to cCR.
\end{abstract}

Keywords: Metastatic breast cancer; anti-HER2 targeted therapy; next-generation sequencing (NGS); adotrastuzumab emtansine (T-DM1); case report

Received: 16 December 2020; Accepted: 30 April 2021; Published: 30 April 2021.

doi: $10.21037 /$ tbcr-21-70

View this article at: http://dx.doi.org/10.21037/tbcr-21-70

\section{Introduction}

Breast cancer has the highest incidence among female malignant tumors worldwide, of which the amplification of human epidermal growth factor receptor 2 (HER2+) occurs in approximately $20 \%$ of patients (1). Before the research and development of the anti-HER2 drugs, the expression of HER2 + in breast cancer implies a poor prognosis. The FDA approval of trastuzumab has placed HER-2-positive breast cancer with remarkably more favorable prognoses. However, some patients would experience disease progression during or after the course of trastuzumabbased treatment. Evidence from several randomized clinical trials strongly suggests that there is a survival benefit from continuing HER2 blockade for metastatic disease in case of progression during or after anti-HER2 targeted therapies (2-5). Ado-trastuzumab emtansine (T-DM1) is an antibody-drug conjugate that is composed of the humanized monoclonal antibody trastuzumab linked to the cytotoxic microtubule inhibitor DM1. It has functions beyond the addiction of the two components, the former selectively and specifically delivers the latter inside the HER2+ cancer cells through receptor-mediated endocytosis. Once released inside the tumor cells, DM1 exerts its cytotoxic effect (6). Currently, T-DM1 is recommended as the second and latter 

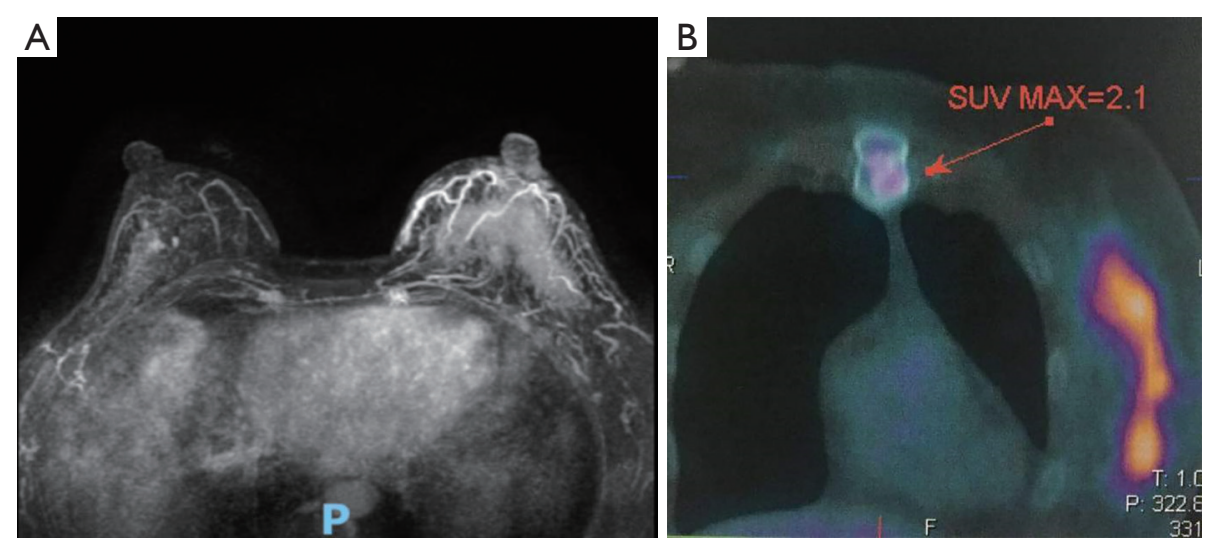

Figure 1 The tumor progressed to skin and sternal metastases during applying the protocol formulated originally. MIP showed a giant mass is significantly enhanced, involving the entire left mammary gland after 3 cycles of epirubicin in combination with cyclophosphamide. (A) PET-CT showed sternal metastasis and its SUVmax was 2.1 after 2 cycles of taxane and trastuzumab. (B). MIP, Maximum Intensity Projection; PET-CT, positron emission tomography-computed tomography.

line of anti-HER2 treatment for the trastuzumab resistance HER2 positive metastatic breast cancer.

Herein we report a patient with HER2-positive metastatic breast cancer who achieved complete clinical remission (cCR) with T-DM1 after progression during several lines of trastuzumab containing regimens therapy. We present the following article in accordance with CARE reporting checklist (available at http://dx.doi.org/10.21037/ tbcr-20-70).

\section{Case presentation}

A 38-years-old woman was diagnosed with T2N0M0, stage IIA invasive carcinoma of the left breast in May 2016. Immunohistochemistry showed ER (-), PR (-), Her2 (3+), Ki67 30\% (+). Her mother was diagnosed with breast cancer at the age of 50. We developed a neoadjuvant chemotherapy regimen that is 4 cycles of epirubicin (epirubicin $90 \mathrm{mg} / \mathrm{m}^{2}$ ) and cyclophosphamide (cyclophosphamide $600 \mathrm{mg} / \mathrm{m}^{2}$ ) followed by 4 cycles of docetaxel and trastuzumab every 3 weeks. When completed 3 cycles of EC, the patient complained of the itch of the left breast. Physical examination showed scattered nodules and sparse erythema in the lateral and central area of the left breast about $10 \times 10 \mathrm{~cm}$ in size, and the erythema spread to the entire left breast after the 4th cycle of EC. Magnetic resonance imaging (MRI) confirmed an increased thickness with irregular and blurred margins involving the skin and the subcutaneous soft tissue of the left breast (Figure 1A). Subsequent skin biopsy confirmed the same molecular subtype as breast lesions. Nevertheless having completed sequential 2 cycles of TH (docetaxel $90 \mathrm{mg} / \mathrm{m}^{2}$, trastuzumab $8 \mathrm{mg} / \mathrm{kg}$ for the first cycle, $6 \mathrm{mg} / \mathrm{kg}$ for the second cycle), the erythema was enlarged, while a PET-CT reported a metastasis in the sternum (Figure 1B). Therefore, dualanti-HER2 therapy comprising trastuzumab, pertuzumab combined with vinorelbine, and cisplatin were administrated in Hongkong. After 3 cycles, the itching eased and the lump in the left breast was softer. But 1 month after interrupting the treatment for personal reasons, the erythema spread to the left armpit apart from the lateral and central areas of the breast subsequently. The treatment was replaced with gemcitabine and cisplatin. Then the next-generation sequencing (NGS) test was performed, that the formalinfixed and paraffin-embedded (FFPE) sample was estimated with a panel of 138 genes, including point mutations, insertions/deletions, gene amplification/loss, gene fusion and rearrangement in and around exons, and gene fusion and rearrangement on partial introns of 10 genes. The result revealed 10 mutations, including 3 copy number variants ERBB2, CDK12, and CCNE1 amplification and 3 point mutations PIK3CA H1047R, TP53 R175H, and MAP3K1 S844Qfs*18. The BRCA was wild type though she had a positive family history (Table 1 ). We added everolimus and trastuzumab the next 2 cycles, and the disease was stable. Then T-DM1 $(160 \mathrm{mg})$ was initiated every 3 weeks in March 2017. Following the first cycle, the skin lesions significantly reduced: the erythema was less extended and the lump touched softer. After 3 cycles of treatment, the lump couldn't be touched. At the end of the 
6th cycle, there was no evident sign on the left breast while the PET-CT SUVmax value of breast lesions decreased to a normal range and the SUVmax value of sternum metastasis was lower than before. The regimen lasted for 19 months and modified to once every 4 weeks subsequently because the whole evaluation reached clinical complete remission (cCR) and lasted for 1 year. After another 10 months, we recommended using T-DM1 every 3 months or interrupting the treatment, the patient chose the latter (Figure 2). During the medication, the patient regularly underwent the PETCT every 3 to 4 months. After stopping the treatment, she postponed the whole evaluation to April 2020 due to the epidemic of COVID-19 and remains free from disease with the latest PET-CT result and physical examination (in April 2020, Figure 3). All procedures performed in studies involving human participants were in accordance with the ethical standards of the institutional and/or national research committee(s) and with the Helsinki Declaration (as

Table 1 Results of all mutations detected by the NGS test

\begin{tabular}{lc}
\hline Genetic mutation & $\begin{array}{c}\text { Abundance of mutation/copy } \\
\text { number }\end{array}$ \\
\hline PIK3CA & $15.70 \%$ \\
H1047R & \\
ERBB2 (HER2) & 46 \\
Gene amplification & \\
TP53 & $33.30 \%$ \\
R175H & \\
MAP3K1 & $9.60 \%$ \\
S844Qfs ${ }^{*} 18$ & \\
CDK12 & 62 \\
Gene amplification & \\
CCNE1 & 15 \\
Gene amplification & \\
\hline
\end{tabular}

NGS, next-generation sequencing. revised in 2013). The patient has given the consent for the case report to be published.

\section{Discussion}

Amplification of human epidermal growth factor receptor 2 (HER2) or HER2 receptor overexpression occurs in approximately $20 \%$ of breast cancer, resulting in worse overall survival without anti-HER2 therapy. According to the recommendation of several international guidelines, anti-HER2 drugs should be contained not only in neoadjuvant or adjuvant therapy but also in salvage treatment $(7,8)$. However, some patients would experience disease progression during or after the course of trastuzumab-based treatment.

The primary trastuzumab resistance is defined as a disease progression within 3 months of first-line trastuzumab treatment in advanced breast cancer and relapse during adjuvant trastuzumab-contained therapy or within 12 months after treatment ends (9). In this case, the tumor progressed during the first 2-cycles of trastuzumabbased therapy, conformed to primary trastuzumab resistance. The HERMINE trial reported that continued using trastuzumab compared to those without trastuzumab treatment could significantly prolong PFS and OS of patients with trastuzumab resistance (10). In the EMILIA trial, T-DM1 demonstrated a better PFS (9.6 vs. 6.4 months respectively; $\mathrm{P}<0.001)$ and $\mathrm{OS}(30.9$ vs. 25.1 months, respectively; $\mathrm{P}<0.001)$ versus lapatinib plus capecitabine after the failure of first-line of anti-HER2 therapy $(4,11)$. Pertuzumab is known as "HER dimerization inhibitors", it can bind site within domain II does not overlap with the epitope on HER2 that is recognized by trastuzumab, and seems to play a key role in overcoming resistance to trastuzumab in the metastatic setting (12). ABC-3 Consensus indicated that trastuzumab plus pertuzumab can be recommended in patients with trastuzumab-resistant

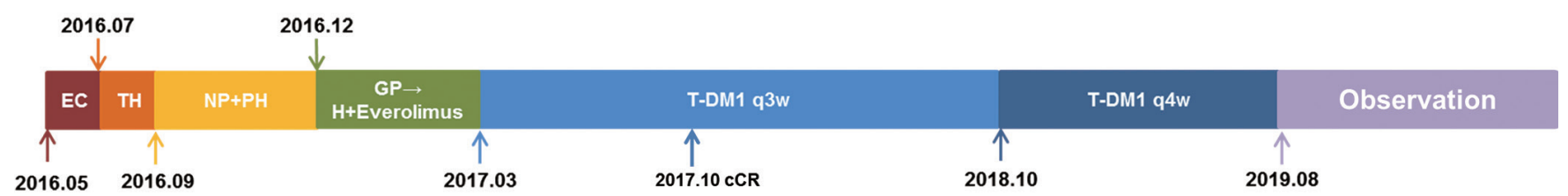

Figure 2 Timeline of the patient's treatments. EC, epirubicin and cyclophosphamide; T, docetaxel; H, trastuzumab; NP, vinorelbine, and platinum; PH, trastuzumab, and pertuzumab; GP, gemcitabine, and platinum; T-DM1, trastuzumab emtansine; q3w, once every 3 weeks; $\mathrm{q} 4 \mathrm{w}$, once every 4 weeks. 


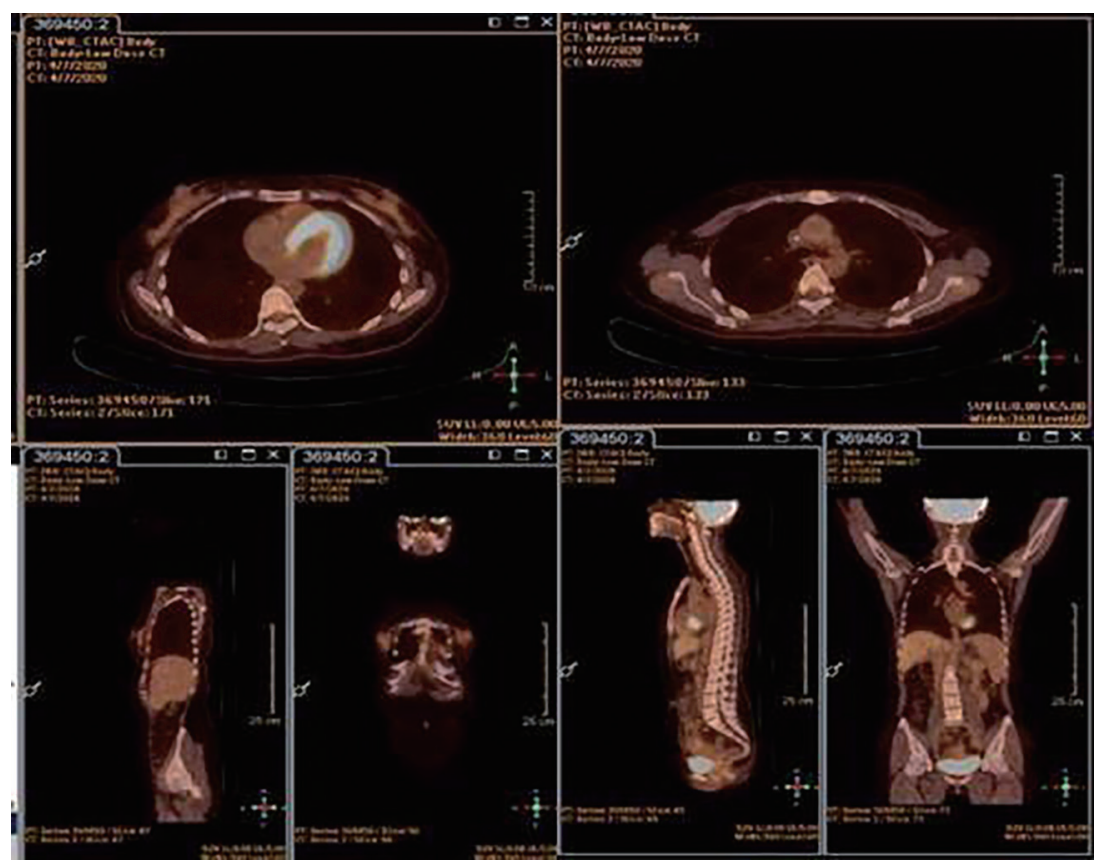

Figure 3 PET-CT result shows maintained clinical complete remission in April 2020. PET-CT, positron emission tomography-computed tomography.

metastatic breast cancer who have not previously received combined HER2-targeting monoclonal antibodies (13). However, the patient went to Hong Kong and received trastuzumab and pertuzumab combined with chemotherapy and the tumor wasn't controlled after 3 cycles of therapy.

A myriad of molecular mechanisms has been postulated to be associated with resistance to trastuzumab, such as disturbance of HER family receptors, (e.g., overexpression of MUC4/MUC1, increased phosphorylation of HER3), parallel receptor pathway activation to bypass HER2 (e.g., upregulation of IGF-1 receptor) and activation on of downstream signaling events distal to HER2 receptor (eg, PIK3CA mutation, mTOR activation) (14).

As is shown in this case, the NGS test detected amplification of HER2, namely anti-HER2 target drugs were still essential and might be effective after the failure of several lines of anti-tumor therapy. However, CCNE1 amplification is a mechanism of trastuzumab resistance in HER2 positive breast cancer, but the exact mechanism is unknown $(15,16)$. We also captured CDK12 amplification that is located at chr17q12 and exhibits high concurrent amplification along with HER2, which accounts for $\sim 90 \%$ of HER2+ breast cancer (17-19). CDK12 reduces susceptibility to trastuzumab and its catalytic activity is mechanistically required for the expression of genes involved in the activation of ErbB-PI3K-AKT cascades (20). MAP3K1 S844Qfs*18 is a kind of rarely documented somatic mutation whose clinical significance is unknown.

Another mutation is PIK3CA p.H1047R. Hyperactivation of the PI3 kinase/Akt/mTOR pathway by aberrant activation of the PIK3CA has been implicated in trastuzumab resistance (21). The patient might benefit from the addition of inhibitors of this pathway according to preclinical models (22). The BOLERO-3 phase III trial showed that patients with trastuzumab resistance advanced breast cancer could benefit from addition of everolimus, one kind of mTOR inhibitor (23). In this case, the tumor reached $\mathrm{SD}$ after receiving trastuzumab with the addition of everolimus plus GP.

T-DM1 is a conjugate combining microtubule inhibitor maytansine (DM1) with trastuzumab. DM1 can destroy the tumor cell microtubules, resulting in cell death, not dependent on the activation status of the PI3K signaling pathway. It not only has the targeting and ADCC effects of trastuzumab but also promotes the cytotoxic drugs exerting their killing effect on tumor cells. The hypothesis was investigated in the randomized phase III EMILIA trial. In this trial, PIK3CA mutation is in connection with shortening PFS and OS in the lapatinib plus capecitabine subgroup, however, the phenomenon has not occurred in patients treated with T-DM1 $(4,11)$. According to these 
results, the ASCO guideline summarizes T-DM1 seems to be effective in both PIK3CA-mutated and PIK3CA wildtype, different from other anti-HER2 therapies.

Besides, another multicenter phase III trial THERESA substantiated that a better median PFS (6.2 vs. 3.3 months, respectively; $\mathrm{P}<0.001)$ and $\mathrm{OS}(22.7$ vs. 15.8 months, respectively; $\mathrm{P}=0.0007)$ were significantly improved by T-DM1 in patients who had undergone progression after at least 2 lines of HER2-targeted regimens for advanced breast cancer than physician's choice $(5,24)$.

The lesions responded to the third line of anti-HER2 treatment with T-DM1 every 3 weeks and reached cCR. Afterward, the frequency was modified to once every 4 weeks and lasted for 10 months. Twenty-two months after achieving cCR, the maintenance treatment was discontinued. Our decision coincides with the ABC-5 Consensus that recommends the optimal duration of maintenance antiHER2 therapy is unknown in patients achieving complete remission. So stopping anti-HER2 therapy after several years of sustained complete remission may be considered in some patients (25). The patient kept cCR condition until April 2020. The cCR of trastuzumab-resistant advanced breast cancer may be associated with the continuous and precise anti-HER2 therapy based on genetic sequencing.

This is a real-world case, showing an excellent efficiency of T-DM1. When several anti-HER2 regimens were inefficient, we formulated the regimen based on the results of gene mutations detected by the NGS test, especially PIK3CA mutations. T-DM1 is one of the standard secondline therapies for advanced trastuzumab-resistant breast cancer. Although the patient applied T-DM1 and achieved cCR, there are still limitations that we should conduct the NGS test after the failure of neoadjuvant chemotherapy as soon as possible.

\section{Conclusions}

We report a case of an HER-2 positive metastatic breast cancer patient who progressed during several lines of chemotherapy and anti-HER2-targeted therapy. The NGS test result revealed mutations and therefore treatment was replaced by T-DM1. The disease achieved cCR and lasted for 30 months even after adjusting the frequency and then discontinuing the medication. This case presents that the long-term disease-free survival was improved by keeping anti-HER2 therapy. What's more, precise treatment based on NGS results can achieve ideal clinical outcomes whose curative effect was disappointing before.

\section{Acknowledgments}

Funding: None.

\section{Footnote}

Reporting Checklist: The authors have completed the CARE reporting checklist. Available at http://dx.doi.org/10.21037/ tbcr-20-70

Conflicts of Interest: All authors have completed the ICMJE uniform disclosure form (available at http://dx.doi. org/10.21037/tbcr-20-70). The authors have no conflicts of interest to declare.

Ethical Statement: The authors are accountable for all aspects of the work in ensuring that questions related to the accuracy or integrity of any part of the work are appropriately investigated and resolved. All procedures performed in studies involving human participants were in accordance with the ethical standards of the institutional and/or national research committee(s) and with the Helsinki Declaration (as revised in 2013). The patient has given the consent for the case report to be published.

Open Access Statement: This is an Open Access article distributed in accordance with the Creative Commons Attribution-NonCommercial-NoDerivs 4.0 International License (CC BY-NC-ND 4.0), which permits the noncommercial replication and distribution of the article with the strict proviso that no changes or edits are made and the original work is properly cited (including links to both the formal publication through the relevant DOI and the license). See: https://creativecommons.org/licenses/by-nc-nd/4.0/.

\section{References}

1. Slamon DJ, Clark GM, Wong SG, et al. Human breast cancer: correlation of relapse and survival with amplification of the HER-2/neu oncogene. Science 1987;235:177-82.

2. Baselga J, Cortés J, Kim SB, et al. Pertuzumab plus trastuzumab plus docetaxel for metastatic breast cancer. $\mathrm{N}$ Engl J Med 2012;366:109-19.

3. Cameron D, Casey M, Press M, et al. A phase III randomized comparison of lapatinib plus capecitabine versus capecitabine alone in women with advanced breast cancer that has progressed on trastuzumab: updated 
efficacy and biomarker analyses. Breast Cancer Res Treat 2008; 112:533-43.

4. Verma S, Miles D, Gianni L, et al. Trastuzumab emtansine for HER2-positive advanced breast cancer. N Engl J Med 2012;367:1783-91. Correction in: N Engl J Med 2013;368:2442.

5. Krop IE, Kim SB, Martin AG, et al. Trastuzumab emtansine versus treatment of physician's choice in patients with previously treated HER2-positive metastatic breast cancer (TH3RESA): final overall survival results from a randomised open-label phase 3 trial. Lancet Oncol 2017;18:743-54.

6. Lewis Phillips GD, Li G, Dugger DL, et al. Targeting HER2-positive breast cancer with trastuzumab-DM1, an antibody-cytotoxic drug conjugate. Cancer Res 2008;68:9280-90.

7. National Comprehensive Cancer Network (NCCN) Clinical Practice Guidelines in Oncology. Breast Cancer. 2016. Available online: https://www.nccn.org/professionals/ physician_gls/default.aspx (accessed July 15, 2016).

8. Cardoso F, Costa A, Norton L, et al. ESO-ESMO 2nd international consensus guidelines for advanced breast cancer (ABC2). Ann Oncol 2014;25:1871-88.

9. Wong $\mathrm{H}$, Leung R, Kwong A, et al. Integrating molecular mechanisms and clinical evidence in the management of trastuzumab resistant or refractory HER-2 ${ }^{+}$metastatic breast cancer. Oncologist 2011;16:1535-46.

10. Extra JM, Antoine EC, Vincent-Salomon A, et al. Efficacy of trastuzumab in routine clinical practice and after progression for metastatic breast cancer patients: the observational Hermine study. Oncologist 2010;15:799-809.

11. Diéras V, Miles D, Verma S, et al. Trastuzumab emtansine versus capecitabine plus lapatinib in patients with previously treated HER2-positive advanced breast cancer (EMILIA): a descriptive analysis of final overall survival results from a randomised, open-label, phase 3 trial. Lancet Oncol 2017;18:732-42. Erratum in: Lancet Oncol 2017;18:e433. Erratum in: Lancet Oncol 2018;19:e667.

12. Zagouri F, Sergentanis TN, Chrysikos D, et al. Pertuzumab in breast cancer: a systematic review. Clin Breast Cancer 2013;13:315-24.

13. Thomssen C, Augustin D, Ettl J, et al. ABC3 Consensus: Assessment by a German Group of Experts. Breast Care (Basel) 2016;11:61-70.

14. Menyhárt O, Santarpia L, Györffy B. A Comprehensive Outline of Trastuzumab Resistance Biomarkers in HER2 Overexpressing Breast Cancer. Curr Cancer Drug Targets 2015;15:665-83.
15. Scaltriti M, Eichhorn PJ, Cortés J, et al. Cyclin $\mathrm{E}$ amplification/overexpression is a mechanism of trastuzumab resistance in HER2+ breast cancer patients. Proc Natl Acad Sci U S A 2011;108:3761-6.

16. Luhtala S, Staff S, Tanner M, Isola J. Cyclin E amplification, over-expression, and relapse-free survival in HER-2-positive primary breast cancer. Tumour Biol. 2016;37:9813-23.

17. Mertins P, Mani DR, Ruggles KV, et al. Proteogenomics connects somatic mutations to signaling in breast cancer. Nature 2016;534:55-62.

18. Sircoulomb F, Bekhouche I, Finetti P, et al. Genome profiling of ERBB2-amplified breast cancers. BMC Cancer 2010;10:539.

19. Cancer Genome Atlas Network. Comprehensive molecular portraits of human breast tumours. Nature 2012;490:61-70.

20. Choi HJ, Jin S, Cho H, et al. CDK12 drives breast tumor initiation and trastuzumab resistance via WNT and IRS1ErbB-PI3K signaling. EMBO Rep 2019;20:e48058.

21. Rimawi MF, De Angelis C, Contreras A, et al. Low PTEN levels and PIK3CA mutations predict resistance to neoadjuvant lapatinib and trastuzumab without chemotherapy in patients with HER2 over-expressing breast cancer. Breast Cancer Res Treat 2018;167:731-40.

22. Ihle NT, Lemos R Jr, Wipf P, et al. Mutations in the phosphatidylinositol-3-kinase pathway predict for antitumor activity of the inhibitor PX-866 whereas oncogenic Ras is a dominant predictor for resistance. Cancer Res 2009;69:143-50.

23. André F, O'Regan R, Ozguroglu M, et al. Everolimus for women with trastuzumab-resistant, HER2-positive, advanced breast cancer (BOLERO-3): a randomised, double-blind, placebo-controlled phase 3 trial. Lancet Oncol 2014;15:580-91.

24. Krop IE, Kim SB, González-Martín A, et al. Trastuzumab emtansine versus treatment of physician's choice for pretreated HER2-positive advanced breast cancer (TH3RESA): a randomised, open-label, phase 3 trial. Lancet Oncol 2014;15:689-99.

25. Cardoso F, Paluch-Shimon S, Senkus E, et al. 5th ESOESMO international consensus guidelines for advanced breast cancer (ABC 5). Ann Oncol 2020;31:1623-49.

doi: $10.21037 /$ tbcr-21-70

Cite this article as: Sun L, Gan L, Wang T. T-DM1 for advanced breast cancer after multi-anti-HER2-target therapy: a case report. Transl Breast Cancer Res 2021;2:15. 